\title{
Upper airway. 3: Sinonasal involvement in chronic obstructive pulmonary disease
}

\author{
$J$ R Hurst
}

Correspondence to:

Dr J R Hurst, Academic Unit of Respiratory Medicine, Royal Free Campus, UCL Medical School, London NW3 2PF, UK; j.hurst@ medsch.ucl.ac.uk

Received 27 April 2009 Accepted 5 May 2009

\section{ABSTRACT}

This review presents the evidence that chronic obstructive pulmonary disease (COPD) is associated with significant sinonasal symptoms, inflammation and airway obstruction. Upper airway symptoms in COPD cause impairment to quality of life. The severity of upper airway involvement relates to that present in the lower airway, suggesting that the nose may be used to model the lung in COPD. More importantly, relationships between upper and lower airway bacteria and inflammation, and the association between sinusitis and treatment failure at exacerbation raise the possibility that nasal intervention in COPD may not only improve health status but may also affect important clinical outcomes such as exacerbation frequency.

It is not widely appreciated that chronic obstructive pulmonary disease (COPD) is associated with sinonasal (upper airway) involvement, a concept that might at first seem surprising given that COPD is defined by the presence of inflammation and airflow limitation in the lung. ${ }^{1}$ However, noxious stimuli to the lung must enter the lower airways via the upper airways, and the airways are in continuity such that this distinction is itself artificial. Additionally, as discussed elsewhere in this series, there are recognised associations between rhinitis and asthma, and these conditions may represent the upper and lower airway manifestations of a single allergic airways disease. This review summarises the evidence supporting sinonasal involvement in COPD, interactions between the upper and lower airway, and the mechanisms and implications that may result from such interaction. Given that COPD is associated with lower airway symptoms, inflammation and airway obstruction, it would seem appropriate to assess whether these features are also present in the upper airway. However, it is important first to consider the effects of cigarette smoke on the nose.

\section{CIGARETTE SMOKE AND THE NOSE}

Unlike asthma-rhinitis, where the nasal airway is probably exposed to a greater allergen load than the lung, most cigarette smoke bypasses the nose and reaches the lung via the mouth. The nasal airway may be exposed to a proportion of the sidestream smoke (which originates from the tip of the cigarette and otherwise escapes to the atmosphere). This contrasts with the lung which predominantly receives mainstream smoke, inhaled through the cigarette itself via the mouth. A proportion of smokers exhale smoke through the nose.

The strongest association between nasal pathology and smoking is for malignant disease (though these tumours are not common), and there is much less evidence that cigarette smoking per se results in a specific nasal inflammatory disease analogous to COPD. ${ }^{2}$ Indeed, surprisingly little is known about non-malignant histological changes in the nose due to cigarette smoke exposure. Nasal pathology does appear to be more common in smokers. ${ }^{3}$ Analysis of the US NHANES II data suggested that smokers have a higher prevalence of upper respiratory tract diagnoses than non-smokers, ${ }^{4}$ whilst in a study of 191 men, current smoking was associated with a fivefold increased risk of chronic rhinitis in a dose-dependent manner. ${ }^{5}$ Nasal mucociliary clearance is prolonged in smokers compared with non-smokers, and longest in smokers who regularly exhale through their nose. ${ }^{6}$ There is also evidence that cigarette smoking is associated with increased nasal resistance.

In summary, although the available data are sparse, it does appear that active cigarette smoking is associated with an increased prevalence of nasal disease and therefore when assessing whether there is sinonasal involvement in COPD appropriate correction for smoking status must be considered.

\section{NASAL SYMPTOMS IN COPD}

This was addressed by one of the first studies ever to investigate the possibility of sinonasal involvement in COPD. ${ }^{8}$ Montnemery et al conducted a large questionnaire-based study from a random selection of a Swedish population in which information on the presence of chronic nasal symptoms and a self-reported diagnosis of COPD was available for 8469 subjects. Thirty-three percent of the total population reported recurrent or persistent nasal symptoms, compared with $40 \%$ of the subjects self-reporting COPD. Recurrent or persistent nasal symptoms were also more prevalent in current smokers than in non-smokers. Although the study is limited by the absence of spirometry to confirm the presence of airflow obstruction, these were the first data to suggest a higher prevalence of nasal symptoms in COPD than in the general population, and a higher prevalence in COPD than in smokers as a whole. In a recent follow-up to the original study, the presence of nasal discharge or nasal blockage in the original survey approximately doubled the risk of a subsequent incident diagnosis of COPD over an 8 year period. ${ }^{9}$ A second epidemiological survey, by van Manen et $a l,{ }^{10}$ investigated the prevalence of self-reported comorbidities in 290 patients with irreversible airflow obstruction and in 421 controls. The prevalence of sinusitis in the controls was $2.5 \%$, compared with $12.4 \%$ in the subjects with airflow obstruction, equivalent to an adjusted odds ratio (OR) of 6 . It is likely that a proportion of the patients with airflow obstruction had chronic asthma rather than COPD. 


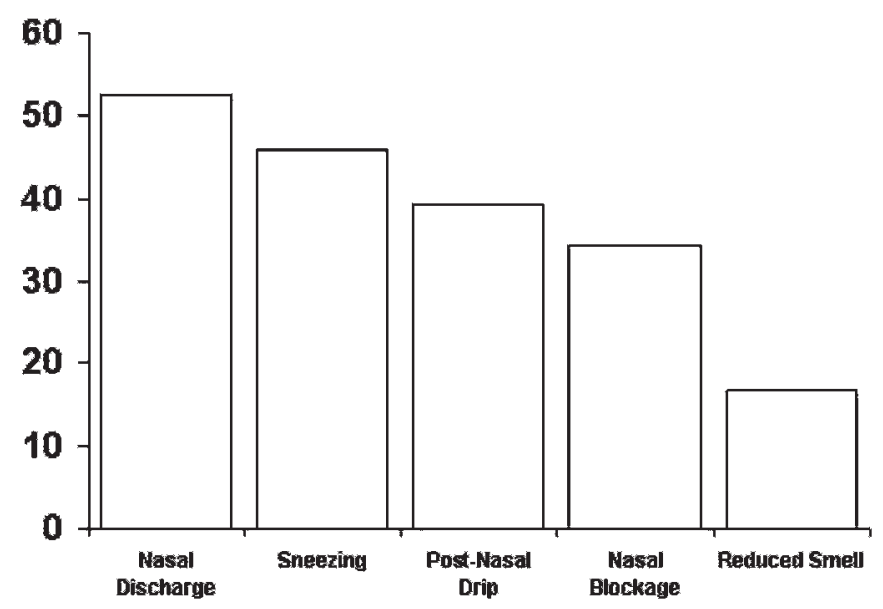

Figure 1 Prevalence (\%) of individual chronic nasal symptoms in 61 patients with stable moderate and severe chronic obstructive pulmonary disease (COPD). Data from Roberts et al, ${ }^{11}$ with permission.

Roberts et $a l^{11}$ were the first to assess the prevalence of chronic nasal symptoms in a population with COPD confirmed at spirometry. In 61 patients with moderate to severe disease (mean forced expiratory volume in $1 \mathrm{~s}\left(\mathrm{FEV}_{1}\right) 0.98$ litre, $37 \%$ predicted), $75 \%$ reported regularly experiencing nasal symptoms when stable, the most common of which was rhinorrhoea (fig 1). Importantly, nasal symptoms were no less prevalent in the ex-smokers, suggesting that nasal symptoms are not solely due to active smoking and that they persist following smoking cessation. Nasal symptoms were also more common in patients with chronic sputum production.

Not all investigators have found an increased prevalence of nasal symptoms in subjects with COPD. A study from Greece compared the prevalence of rhinitis in subjects with and without COPD. ${ }^{12}$ COPD was confirmed with spirometry, and rhinitis was defined by the presence of nasal symptoms together with objective evidence of reduced nasal airflow at rhinomanometry. Of the 6112 subjects assessed, $6 \%$ had COPD and $25 \%$ had rhinitis. The prevalence of rhinitis was higher in smokers than in both ex-smokers and never-smokers (30, 27 and 20\%, respectively), but the prevalence of rhinitis in COPD (28\%) was not significantly different from the prevalence in subjects without COPD (25\%). Notably, the patients with COPD in this study were likely to have had milder disease than those studied by Roberts et al. ${ }^{11}$

In conclusion, it seems likely that COPD is associated with a higher prevalence of chronic nasal symptoms than found in control subjects, not solely attributable to active cigarette smoking and which persist after smoking cessation. The presence of chronic nasal symptoms suggests that COPD may be associated with chronic rhinosinusitis. Difficulties in assessing sinonasal disease using symptoms alone has led national ${ }^{13}$ and internationa $\mathrm{l}^{14}$ guideline committees additionally to require objective evidence of disease on physical examination or imaging studies. To date there are no reports of sinonasal imaging in COPD, but further evidence of sinonasal involvement has been provided by studies of nasal inflammation. These are discussed further in the following section.

\section{NASAL INFLAMMATION IN COPD}

A number of studies have now demonstrated that stable COPD is associated with increased nasal inflammation, and these are summarised in table 1.

The first study to assess nasal inflammation in COPD was published in 2003. Nihlen et a l $^{15}$ performed nasal lavage before and after histamine challenge in 23 patients with COPD and 26 controls, each group containing both current smokers and never- or ex-smokers. Although there were no differences in the concentrations of inflammatory markers between the subjects with COPD and controls at baseline, prior to the histamine challenge, this probably reflected the methodology employed in which initial nasal wash samples were discarded. Histamine challenge resulted in a greater nasal neutrophil response in the subjects with COPD compared with the controls (assessed using myeloperoxidase), greatest in the subjects with COPD reporting nasal symptoms who also had an increase in nasal fucose concentration (a marker of mucinous secretion). The authors concluded that "individuals suffering from both COPD and nasal complaints exhibited greater nasal neutrophil activity and secretory responsiveness than COPD patients without nasal symptoms". ${ }^{15}$ The study is open to criticism regarding the inclusion of current, ex-smokers and never-smokers (thus being unable to

Table 1 Studies investigating nasal inflammation in stable COPD

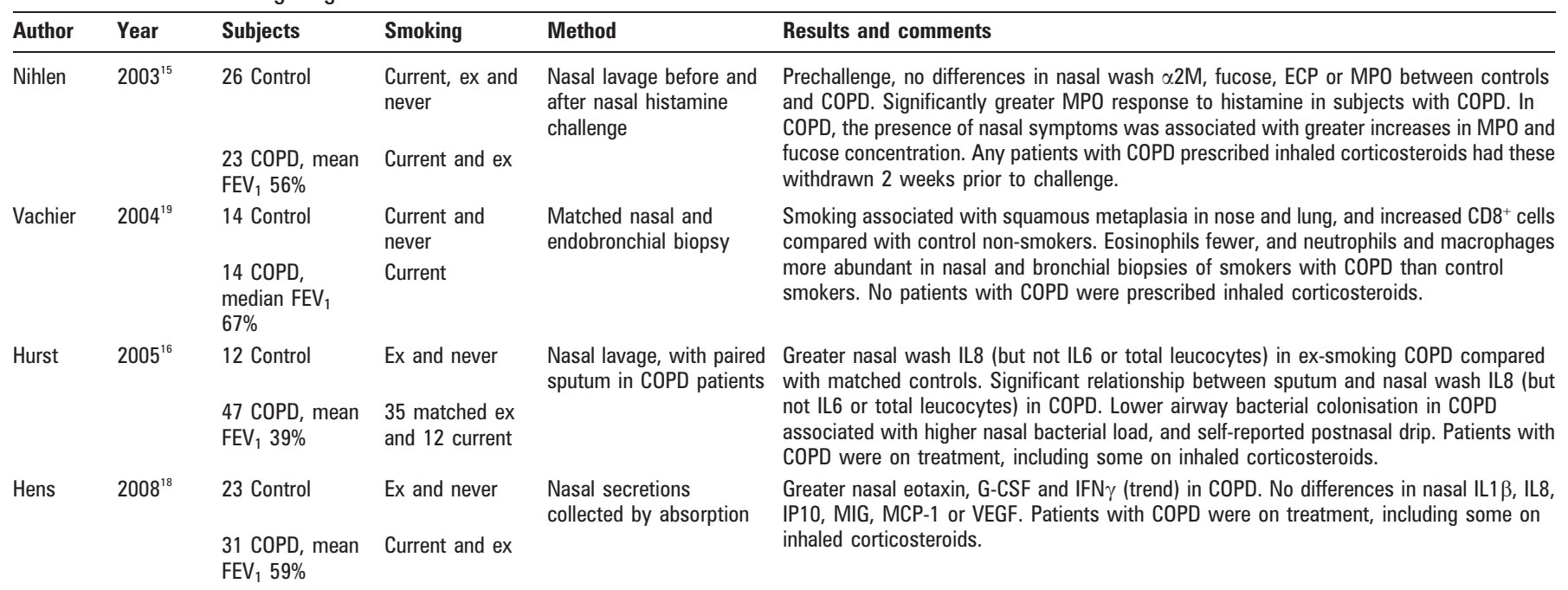

COPD, chronic obstructive pulmonary disease; ECP, eosinophil cationic protein; FEV 1 , forced expiratory volume in $1 \mathrm{~s}$; G-CSF, granulocyte colony-stimulating factor; IFN $\gamma$, interferon $\gamma_{;}$IL, interleukin; IP-10, interferon $\gamma$-induced protein 10; $\alpha 2 \mathrm{M}, \alpha$-2-macroglobulin; MIG, monokine-induced by IFN $\gamma$; MCP-1, monocyte chemotactic protein-1; MPO, myeloperoxidase; VEGF, vascular endothelial growth factor. 


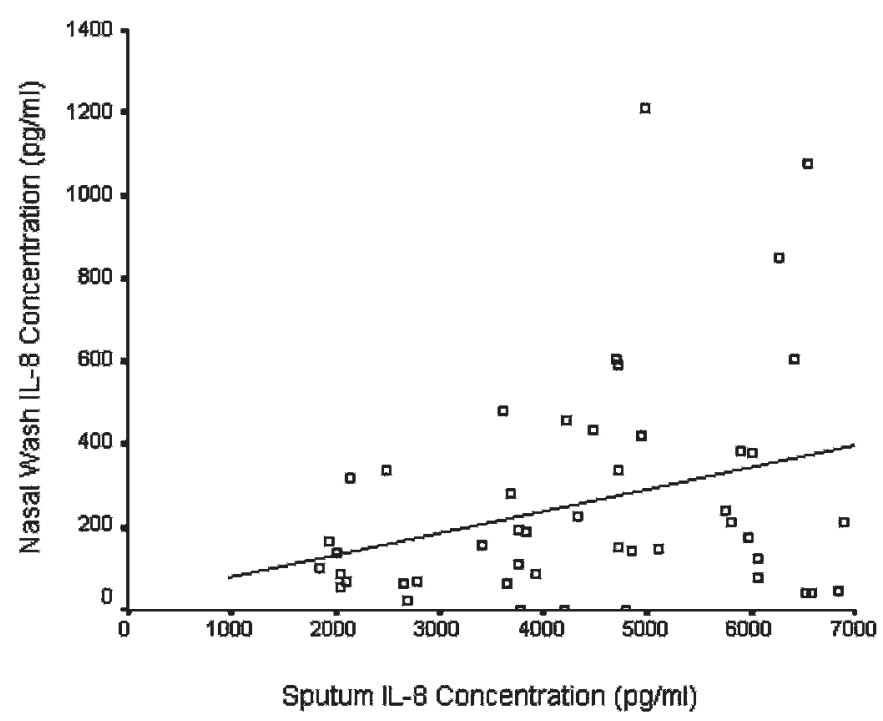

Figure 2 The magnitude of the nasal inflammatory response in stable chronic obstructive pulmonary disease (COPD) relates to that occurring in the lung: correlation between interleukin 8 (IL8) concentration in paired nasal wash and sputum samples from 47 patients with COPD $(r=0.30$, $\mathrm{p}=0.039$ ). From Hurst $e t a{ }_{1}{ }^{16}$ with permission.

differentiate any inflammation caused by current smoking from that associated with COPD), and the experimental design which probably biased against detecting differences between subjects with COPD and control subjects in the baseline state.

We developed this initial description of nasal inflammation in COPD by performing a study that included a smoking-matched control group, and which additionally assessed relationships between nasal and bronchial inflammation. ${ }^{16}$ Even following smoking cessation, COPD was associated with greater nasal inflammation than that present in an ex-smoking or neversmoking control group of similar age and sex distribution. Moreover, the degree of nasal inflammation reflected that present in the lung, as evidenced by a significant correlation between the concentration of interleukin 8 (IL8) in matched nasal wash and sputum samples (fig 2). Bacterial colonisation is an important phenomenon in COPD, and patients with lower airway colonisation both had a higher total nasal bacterial load and were more likely to experience postnasal drip. Similarities between upper and lower airway inflammation in COPD are therefore also associated with relationships between upper and lower airway bacterial carriage. Eradicating upper airway pathogens in the critical care setting is associated with a reduced risk of ventilator-associated pneumonia, ${ }^{17}$ and such an approach deserves investigation in COPD.

Hens et al recently reported a study enrolling fewer patients, but which assessed a greater number of nasal inflammatory markers. ${ }^{18}$ Patients with COPD had higher nasal concentrations of eotaxin, granulocyte colony-stimulating factor (G-CSF), and a trend to higher interferon $\gamma$ concentrations than control subjects, without significant differences in IL1 $\beta$, IL8, IP10 (interferon $\gamma$-inducible protein 10), MIG (monokine induced by interferon $\gamma$ ), MCP-1 (monocyte chemoattractant protein-1) or VEGF (vascular endothelial growth factor). This study did not control for the effects of active cigarette smoking, and with multiple comparisons in a small group of subjects there is the attendant risk of type I and type II errors. However, this is also the only study to date that has reported nasendoscopy findings in patients with COPD. There were more signs of nasal inflammation in the patients with COPD than in the controls, though the assessor was not blinded to the presence or absence of disease. As with the study by Hurst et al, ${ }^{16}$ the concentrations of inflammatory markers in the nose were not significantly related either to indices of airflow obstruction or to the severity of nasal symptoms.

Vachier et al ${ }^{19}$ took a different approach and assessed paired nasal and bronchial biopsies in smokers with and without COPD, and non-smoking controls. The patients with COPD had mild disease with a median $\mathrm{FEV}_{1}$ of $67 \%$ predicted, none had nasal symptoms and none was prescribed inhaled corticosteroids. Smoking was associated with squamous metaplasia and infiltration of the nasal and bronchial mucosa with $\mathrm{CD} 8^{+}$cells. There were differences between the smokers with and without COPD in the number of eosinophils (fewer in COPD), neutrophils and macrophages (both more abundant in smokers with COPD), suggesting that COPD is associated with further increases in nasal inflammation over that due to smoking alone. The study did not report on relationships between the relative severity of upper and lower airway inflammation in the subjects with COPD.

These four studies 15161819 are the only reports to date that have examined the presence of sinonasal inflammation in stable COPD. However, approaching the question from a rhinological perspective, two studies by Ragab et al also warrant discussion. ${ }^{20}{ }^{21}$ These examined lower airway involvement in a small number of patients with chronic rhinosinusitis who had failed medical treatment. Sixty percent were found to have lower airway symptoms or spirometric abnormalities (though the methodology does not permit a distinction between asthma and COPD). ${ }^{20}$ Moreover, patients who had asymptomatic airflow obstruction had a higher proportion of neutrophils in sinus washings than those with normal spirometry, and the number of these neutrophils correlated with the degree of airflow obstruction. ${ }^{21}$ This provides further evidence of relationships between the degree of upper and lower airway involvement in these patients, though the results are difficult to interpret with specific regard to COPD.

Exacerbations of COPD are important events resulting in considerable morbidity and mortality. Exacerbations are associated with further increases in both lower airway and systemic inflammation, ${ }^{22}$ and there are also data examining nasal inflammatory changes at exacerbation. In a small pilot study of experimental rhinovirus-induced exacerbations, there was an increase in nasal IL8 concentration over baseline which peaked on day 4 following inoculation, coinciding with the peak in nasal viral load. ${ }^{23}$ Interestingly, patients with COPD needed a very low dose of virus to become infected, and it is tempting to attribute this to increased nasal inflammation. The major subtype of rhinovirus enters cells through association with intercellular adhesion molecule-1 (ICAM-1), a receptor that may be upregulated in response to inflammation. In support of this hypothesis, patients prone to frequent COPD exacerbations have greater airway inflammation in the stable state ${ }^{24}$ and are also more susceptible to developing colds. ${ }^{25}$ Only one study has investigated nasal inflammation in naturally occurring exacerbations. ${ }^{22}$ We reported that markers of nasal inflammation were higher in patients at exacerbation than at baseline, and (as at baseline) there was a significant correlation between the degree of upper and lower airway inflammation. The presence of nasal rhinovirus was associated with a higher nasal wash IL6 concentration than that present in non-rhinoviral exacerbations, raising the possibility that nasal wash indices may prove useful biomarkers of exacerbation aetiology. In contrast to the baseline state, at exacerbation there were significant correlations between the severity of nasal symptoms and the nasal wash concentrations of both IL6 and IL8. 
In summary, there is accumulating evidence that patients with COPD have upregulated nasal inflammation compared with controls, additive to that associated with current cigarette smoking. This inflammation persists after smoking cessation, and the magnitude correlates with that present in the lower airway. There are also relationships between upper and lower airway bacterial carriage. Nasal inflammation is further upregulated at exacerbation, and the magnitude of the nasal inflammatory response at exacerbation relates to the severity of nasal symptoms.

\section{NASAL AIRWAY OBSTRUCTION IN COPD}

COPD is defined by the presence of airflow obstruction in the lung ${ }^{1}$ and it is therefore plausible that sinonasal involvement in COPD might result in obstruction to the nasal airway. Although the mucosa of the nasal and pulmonary airways are similar, both formed of pseudostratified columnar ciliated epithelium, there are significant differences in the submucosa, and this has important implications for the generation of airway narrowing. Airway obstruction in the nose is predominantly caused by engorgement of venous sinusoids, in comparison with the lung where smooth muscle tone, thickening of the airway wall and excess mucus are major contributors.

We have assessed nasal airway obstruction in COPD using acoustic rhinometry, ${ }^{26}$ a non-invasive technique in which a reflected sound pulse is used to model nasal cross-sectional area as a function of distance into the nose. It is important to remark that acoustic rhinometry measures nasal airway rather than airflow obstruction, and that there are at present no reports of changes in nasal airflow in patients with COPD. The portion of the nasal airway most susceptible to inflammatory obstruction can be recognised on a rhinometry trace as the second minimum cross-sectional area (MCA2). We have reported a significantly lower MCA2, reflecting a narrower nasal airway, in patients with COPD experiencing chronic nasal symptoms compared with those without such symptoms. ${ }^{26}$ There was also a significant correlation between the severity of nasal airway and pulmonary airflow obstruction, ${ }^{26}$ the magnitude of which was similar to that described between upper and lower airway IL8 concentrations. ${ }^{16}$ This therefore provides further evidence that nasal involvement in COPD is similar in effect and magnitude to that occurring in the lung.

\section{MECHANISMS OF INTERACTION BETWEEN THE UPPER AND LOWER AIRWAY IN COPD}

Taken together, the evidence presented above demonstrates that patients with COPD have more nasal symptoms than controls, which persist after smoking cessation. There is also a nasal inflammatory response in COPD, the severity of which relates to the severity of lower airway inflammation. Similarities in upper and lower airway involvement are further reflected by a relationship between the severity of pulmonary airflow obstruction and obstruction to the nasal airway. Therefore, there seems little doubt that COPD is associated with the coexistence of nasal symptoms, airway obstruction and inflammation. However, an important point remains: does sinonasal involvement in COPD simply represent coexistent disease or can the presence of inflammation at one site in the airway affect outcomes at another? If true, this would lead to the hypothesis that nasal intervention in COPD could affect important lower airway outcomes such as lung function decline or exacerbations.
How might nasal involvement in COPD affect the lower airway? First, loss of the normal conditioning function of the nose will allow unfiltered, cold and dry air to reach the bronchi. Secondly, elegant work in asthma-rhinitis has demonstrated that application of an inflammatory stimulus to the nasal mucosa results in lower airway inflammation, ${ }^{27}$ and vice versa, ${ }^{28}$ mechanisms that are likely to operate via the systemic (blood) compartment such that there may be "homing" of inflammatory cells to the entire airway in response to inflammatory stimulation at any part of it. Thirdly, bronchoconstrictor neuronal responses may play a role, though the existence of nasobronchial reflexes remains controversial. ${ }^{29}$ Finally, the relationship between postnasal drip and lower airway bacterial colonisation $^{16}$ suggests that direct passage of mediators or pathogens along the respiratory mucosa may be important. These concepts are summarised in fig 3 .

\section{CLINICAL IMPLICATIONS OF NASAL INVOLVEMENT IN COPD}

Two studies, in different populations, have now reported that the presence of nasal symptoms in COPD is associated with impairment to quality of life. ${ }^{18}{ }^{30}$ Both employed variants of the Sino-Nasal Outcome Test (SNOT) questionnaire, a validated disease-specific health status tool. The study of Hens et a ${ }^{18}$ included a control population and showed that SNOT scores were significantly worse in the subjects with COPD. We examined relationships between the SNOT score and the St. George's Respiratory Questionnaire, ${ }^{30}$ reporting a weak and statistically non-significant correlation. One explanation for this could be that the total health status burden in COPD is not fully reflected using tools that do not include an upper airway component.

The only current data relating clinically important outcomes in COPD to sinonasal involvement come from a study investigating "treatment failure" at exacerbation. ${ }^{31}$ Treatment failure was defined as the need for a further physician visit with persistent respiratory symptoms requiring a change in antibiotics. A total of 232 exacerbations in 107 patients were studied

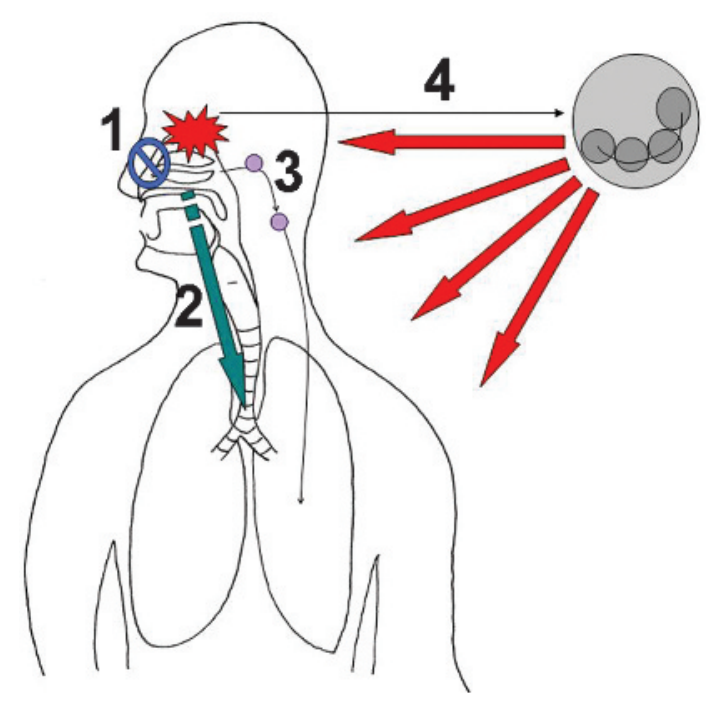

Figure 3 Potential mechanisms for interaction between the upper and lower airways in chronic obstructive pulmonary disease (COPD). (1) Loss of nasal conditioning function. (2) Direct passage of inflammatory mediators and/or microorganisms between the upper and lower respiratory tract. (3) Nasobronchial neuronal reflexes. (4) Stimulation at one point of the respiratory mucosal surface results in a pan-airway inflammatory response. 
and the failure rate was $15 \%$. Intriguingly, a history of sinusitis was significantly more likely to be present in the patients who experienced one or more treatment failures than in those whose exacerbations always recovered (29\% vs 9\%, $p=0.009)$. A history of sinusitis was not associated with the presence of any of the other five variables that predicted treatment failure (exacerbation frequency, disease severity, the need for home oxygen therapy, the prescription of maintenance oral corticosteroids or a prior history of pneumonia).

These two findings - that nasal symptoms are associated with health status impairment and that the presence of sinusitis is associated with treatment failure at exacerbation-raise the hypothesis that therapeutic nasal intervention might be a novel approach to therapy in COPD. An effective nasal intervention might not only reduce nasal symptoms, and thereby improve health status, but also affect clinically important lower airway outcomes such as exacerbation frequency. At present there are no trials reporting nasal interventions in COPD. Indeed, whilst the nasal route is commonly used to deliver oxygen and administer non-invasive ventilation, there are also no studies investigating relationships between nasal inflammation and use of these treatments.

A final and attractive implication of pan-airway involvement in COPD is the opportunity to use the nose to study the lung. This is particularly important in patients with more severe COPD where it becomes difficult to justify elective endobronchial biopsy. Obtaining nasal biopsies is much less invasive, can be repeated more readily, and similarities between the pathological findings in COPD nasal and bronchial biopsies have been described above. ${ }^{19}$ A recent study has also demonstrated that cell surface marker expression and functional responses of matched, cultured nasal and bronchial cells are similar, ${ }^{32}$ and some of the subjects recruited to this study had COPD. This builds on earlier work demonstrating that nasal and bronchial epithelial cell cultures are of similar size and shape, and have similar growth rates and ciliary beat frequency. ${ }^{33}$ Gene expression alterations in relation to smoking are also similar in bronchial and nasal mucosa, ${ }^{34}$ though a small study in 20 subjects examining the presence of sputum DNA microsatellite instability failed to find such changes simultaneously in the nose. ${ }^{35}$

\section{AREAS FOR FUTURE STUDY}

Although there is now considerable evidence from multiple sources reporting nasal symptoms, and inflammatory and functional changes in COPD, the individual studies are relatively small and there is still the need for a definitive, larger investigation of sinonasal involvement in COPD. This should be performed across the spectrum of COPD disease severity, and comprehensively assess nasal symptoms, examination findings, inflammation, bacterial carriage, sinonasal imaging and function.

The second major unanswered question is whether it is possible to affect sinonasal involvement in COPD with nasal treatment-aiming to reduce nasal symptoms, improve quality of life and affect important lower airway outcomes such as exacerbations.

\section{CONCLUSIONS}

COPD can be associated with upper airway (sinonasal) symptoms that affect quality of life, and a nasal inflammatory process. This is not, at present, reflected in COPD guidelines. Practioners in otolaryngology might consider the diagnosis of
COPD in patients with chronic rhinosinusitits, and practioners in respiratory medicine should consider identifying those COPD patients with chronic nasal symptoms. In the absence of specific intervention studies in COPD, nasal symptoms should currently be managed by reference to appropriate guidelines. ${ }^{13}$ For those considering intervention studies, guidelines exist for the conduct of such research, ${ }^{36}$ and it must be hoped that such studies are indeed performed given the urgent need for truly novel treatment strategies to treat this prevalent and devastating disease.

Competing interests: None.

Provenance and peer review: Commissioned; not externally peer reviewed.

\section{REFERENCES}

1. Global Strategy for Diagnosis, Management and Prevention of COPD Available at: http://www.goldcopd.com/GuidelinesResources.asp (accessed 7 Apr 2009).

2. Phillips DE, Hill L, Weller $P$, et al. Tobacco smoke and the upper airway. Clin Otolaryngol Allied Sci 2003;28:492-6.

3. Benninger MS. The impact of cigarette smoking and environmental tobacco smoke on nasal and sinus disease: a review of the literature. Am J Rhinol 1999;13:435-8.

4. Turkeltaub PC, Gergen PJ. Prevalence of upper and lower respiratory conditions in the US population by social and environmental factors: data from the second National Health and Nutrition Examination Survey, 1976 to 1980 (NHANES II). Ann Allergy 1991;67:147-54.

5. Annesi-Maesano I, Oryszczyn MP, Neukirch F, et al. Relationship of upper airway disease to tobacco smoking and allergic markers: a cohort study of men followed up for 5 years. Int Arch Allergy Immunol 1997;114:193-201.

6. Stanley PJ, Wilson R, Greenstone MA, et al. Effect of cigarette smoking on nasal mucociliary clearance and ciliary beat frequency. Thorax 1986;41:519-23.

7. Dessi P, Sambuc R, Moulin G, et al. Effect of heavy smoking on nasal resistance. Acta Otolaryngol 1994;114:305-10.

8. Montnemery P, Svensson C, Adelroth E, et al. Prevalence of nasal symptoms and their relation to self-reported asthma and chronic bronchitis/emphysema. Eur Respir J 2001;17:596-603.

9. Nihlen U, Montnemery P, Andersson M, et al. Specific nasal symptoms and symptom-provoking factors may predict increased risk of developing COPD. Clin Physiol Funct Imaging 2008;28:240-50.

10. van Manen JG, Bindels PJE, ljzermans CJ, et al. Prevalence of comorbidity in patients with a chronic airway obstruction and controls over the age of 40. J Clin Epidemiol 2001;54:287-93.

11. Roberts NJ, Lloyd-Owen SJ, Rapado F, et al. Relationship between chronic nasal and respiratory symptoms in patients with COPD. Respir Med 2003;97:909-14.

12. Sichletidis L, Tsiotsios I, Gavriilidis A, et al. Prevalence of chronic obstructive pulmonary disease and rhinitis in Northern Greece. Respiration 2005;72:270-7.

13. Scadding GK, Durham SR, Mirakian R, et al. BSACl guidelines for the management of rhinosinusitis and nasal polyposis. Clin Exp Allergy 2008;38:260-75.

14. Benninger MS, Ferguson BJ, Hadley JA, et al. Adult chronic rhinosinusitis: definitions, diagnosis, epidemiology and pathophysiology. Otolaryngol Head Neck Surg 2003;129(Suppl):S1-32.

15. Nihlen U, Andersson M, Lofdahl CG, et al. Nasal neutrophil activity and mucinous secretory responsiveness in COPD. Clin Physiol Funct Imaging 2003;23:138-42.

16. Hurst JR, Wilkinson TM, Perera WR, et al. Relationships among bacteria, upper airway, lower airway, and systemic inflammation in COPD. Chest 2005;127:1219-26.

17. de Smet AM, Kluytmans JAJW, Cooper BS, et al. Decontamination of the digestive tract and oropharynx in ICU patients. N Engl J Med 2009;360:20-31.

18. Hens G, Vanaudenaerde BM, Bullens DMA, et al. Sinonasal pathology in nonallergic asthma and COPD: "united airway disease" beyond the scope of allergy. Allergy 2008;63:261-7

19. Vachier I, Vignola AM, Chiappara G, et al. Inflammatory features of nasal mucosa in smokers with and without COPD. Thorax 2004;59:303-7.

20. Ragab A, Clement $P$, Vincken W. Objective assessment of lower airway involvement in chronic rhinosinusitis. Am J Rhinol 2004;18:15-21.

21. Ragab A, Clement P, Vincken W. Correlation between the cytology of the nasal middle meatus and BAL in chronic rhinosinusitis. Rhinology 2005;43:11-7.

22. Hurst JR, Perera WR, Wilkinson TM, et al. Systemic and upper and lower airway inflammation at exacerbation of chronic obstructive pulmonary disease. Am J Respir Crit Care Med 2006;173:71-8.

23. Mallia P, Message SD, Kebadze T, et al. An experimental model of rhinovirus induced chronic obstructive pulmonary disease exacerbations: a pilot study. Respir Res 2006;7:116.

24. Bhowmik A, Seemungal TA, Sapsford RJ, et al. Relation of sputum inflammatory markers to symptoms and lung function changes in COPD exacerbations. Thorax 2000:55:114-20.

25. Hurst JR, Donaldson GC, Wilkinson TM, et al. Epidemiological relationships between the common cold and exacerbation frequency in COPD. Eur Respir $J$ 2005;26:846-52. 
26. Hurst JR, Kuchai R, Michael P, et al. Nasal symptoms, airway obstruction and disease severity in chronic obstructive pulmonary disease. Clin Physiol Funct Imaging 2006;26:251-6.

27. Braunstahl GJ, Overbeek SE, Kleinjan A, et al. Nasal allergen provocation induces adhesion molecule expression and tissue eosinophilia in upper and lower airways. J Allergy Clin Immunol 2001;107:469-76.

28. Braunstahl GJ, Overbeek SE, Fokkens WJ, et al. Segmental bronchoprovocation in allergic rhinitis patients affects mast cell and basophil numbers in nasal and bronchial mucosa. Am J Respir Crit Care Med 2001;164:858-65.

29. Sarin S, Undem B, Sanico A, et al. The role of the nervous system in rhinitis. J Allergy Clin Immunol 2006;118:999-1016.

30. Hurst JR, Wilkinson TM, Donaldson GC, et al. Upper airway symptoms and quality of life in chronic obstructive pulmonary disease (COPD). Respir Med 2004;98:767-70
31. Dewan NA, Rafique S, Kanwar B, et al. Acute exacerbation of COPD. Factors associated with poor treatment outcome. Chest 2000;117:662-71.

32. McDougall CM, Blaylock MG, Douglas JG, et al. Nasal epithelial cells as surrogates for bronchial epithelial cells in airway inflammation studies. Am J Respir Cell Mol Biol 2008;39:560-8.

33. Devalia JL, Sapsford RJ, Wells CW, et al. Culture and comparison of human bronchial and nasal epithelial cells in vitro. Respir Med 1990;84:303-12.

34. Sridhar S, Schembri F, Zeskind J, et al. Smoking-induced gene expression changes in the bronchial airway are reflected in nasal and buccal epithelium. BMC Genomics 2008;9:259.

35. Karatzanis AD, Samara KD, Tzortzaki E, et al. Microsatellite DNA instability in nasa cytology of COPD patients. Oncol Rep 2007;17:661-5.

36. Meltzer E0, Hamilos DL, Hadley JA, et al. Rhinosinusitis: developing guidance for clinical trials. J Allergy Clin Immunol 2006;118(Suppl):S17-61.

\section{Pulmonary puzzle}

\section{ANSWER}

From the question on page 56

The transbronchial biopsy showed large numbers of mononuclear cells in both bronchial and parenchymal tissue. The mononuclear cells were heavily parasitised with Leishmania amastigotes (figs 1 and 2). No granulomas were seen. Leishmania spp. were also identified in a parotid aspirate (Supplementary fig 4). Stains and cultures were negative for bacteria, fungi, mycobacteria, Pneumocystis jirovecii and cytomegalovirus.

Leishmaniasis is usually spread through sandfly bites, although direct human-human transmission may occur via needle sharing. ${ }^{2}$ The Leishmania parasite resides free in the sandfly digestive tract in the proamastigote form. Upon entering the human host, the parasite is taken up by macrophages where it transforms to the obligate intracellular amastigote stage. Depending on the leishmaniasis species, macrophage traffic patterns and host immunity, the infection may remain localised in the skin (cutaneous leishmaniasis) or may disseminate systemically (visceral leishmaniasis or kalaazar as in this case). The typical systemic spread is throughout the reticuloendothelial system (bone marrow, spleen and liver) although any organ may be affected. Pulmonary involvement with kala-azar is uncommon, with very few cases reported. ${ }^{3}$ Diagnosis is usually made by biopsy of the affected organs, although microbiological, serological and PCR techniques exist. Visceral leishmaniasis is treated with pentavalent antimony compounds or amphotericin B. ${ }^{4}$

Patients with advanced HIV disease frequently present with respiratory illness, and the differential diagnosis is extremely wide. ${ }^{5}$ Asymptomatic hepatosplenomegaly is a common feature in patients with HIV, although in this clinical context its presence narrows the differential somewhat to cytomegalovirus, toxoplasmosis, leishmaniasis, schistosomiasis, endemic mycoses, $M$ avium complex, miliary tuberculosis and HIV-associated lymphoma. The previous treatment for leishmaniasis was a "red-herring" since the parasite is difficult to eradicate completely and may recur, especially in immunosuppressed patients.

\section{REFERENCES}

1. Piscopo TV, Mallia AC. Leishmaniasis. Postgrad Med J 2006;82:649-57.

2. Cruz I, Morales MA, Noguer I, et al. Leishmania in discarded syringes from intravenous drug users. Lancet 2002;359:1124-5.

3. Sarker CB, Chowdhury KS, Siddiqui NI, et al. Clinical profile of Kala-azar in adults: as seen in Mymensingh Medical College Hospital, Mymensingh, Bangladesh. Mymensingh Med J 2003:12:41-4.

4. Alvar J, Aparicio P, Aseffa A, et al. The relationship between leishmaniasis and AIDS: the second 10 years. Clin Microbiol Rev 2008;21:334-59.

5. Huang L, Stansell J. Pulmonary complications of human immunodeficiency virus infection. In: Mason RJ, Broaddus VC, Murray JF, et al, eds. Murray and Nadel's textbook of respiratory medicine. 4th edn. Philadelphia, PA: Elsevier Saunders, 2005:2111-62. 